\title{
Developmental Robotics and its Role Towards Artificial General Intelligence
}

\author{
Manfred Eppe ${ }^{1} \cdot$ Stefan Wermter ${ }^{1} \cdot$ Verena V. Hafner $^{3} \cdot$ Yukie Nagai $^{2}$
}

Accepted: 13 January 2021 / Published online: 14 February 2021

(c) The Author(s) 2021

Human intelligence develops through experience, robot intelligence is engineered - is it? At least in the mainstream approaches based on classical Artificial Intelligence (AI) and Machine Learning (ML) the robotic engineering approach is pursued and data- or knowledge-based algorithms are designed to improve a robot's problem-solving performance. Based on this engineering perspective of classical $\mathrm{AI} / \mathrm{ML}$ approaches plenty of valuable application-specific impact has been achieved. Yet, the achievements are often subject to restrictions that involve domain knowledge as well as constraints concerning application domains and computational hardware.

Developmental Robotics seeks to extend this constrained perspective of engineered artificial robotic cognition by building on inspiration from biological, developmental processes to design robots that learn in an open-ended continuous fashion. Herein, Developmental Robotics seeks to investigate and model cognitively plausible inductive biases including curiosity, homeostasis and body schemas. This enables Developmental Robotics to consider cognitive domains that involve problem-solving, self-perception, developmental disorders and embodied cognition.

This cognitive modeling perspective helps to improve the performance of intelligent robotic agents, and it has already led to significant contributions that inspired cutting-edge application-oriented Machine Learning technology. In addition, Developmental Robotics also provides functional

Manfred Eppe

eppe@informatik.uni-hamburg.de

1 Department of Informatics, Universität Hamburg, Vogt-Kölln-Str. 30, 22527 Hamburg, Germany

2 International Research Center for Neurointelligence, University of Tokyo, 7-3-1 Hongo, Bunkyo-ku, Tokyo 113-0033, Japan

3 Department of Computer Science, Humboldt-Universität zu Berlin, Unter den Linden 6, 10099 Berlin, Germany computational models that help to understand and to investigate embodied cognitive processes.

In this special issue we collect and address several of the challenges that adhere to the modeling of inductive biases and developmental robots. In their review, Nguyen et al. [4] provide an overview over the state of the art in modeling the body schema and peripersonal space of robotic agents. The authors conclude by proposing a novel theoretical model to learn the peripersonal space and body schema representations. Other technical contributions include research by Hoffman et al. [3], who propose a computational model of the well-known mirror self-recognition test that is deemed to indicate whether animals (e.g. dolphins or apes) are selfaware. The authors deploy the model on a humanoid robot and show that if the test indeed indicates self-awareness for biological entities, then their computational robotic implementation also shows self-awareness. Furthermore, Philippsen [6] investigates developmental models of speech acquisition, and in a project report, Nguyen et al. [5] summarize their recent work on curriculum learning and continual learning for robots. The continual learning aspect has also been picked up by Schillaci et al. [7]. The authors show that the computational models for continual learning do not only improve robots and deliver empirical foundations to better understand intelligent behavior. They are also useful for practical applications outside of the robotics domain, such as greenhouse models for horticulture applications.

Controversial discussions and perspectives are provided by Pierre-Yves Oudeyer [2] and Butz [1]. We have interviewed Pierre-Yves Oudeyer and asked him for his perspective on strong and weak AI. He argues that the terminology of weak and strong is misleading because it implicitly assumes a scale of intelligent behavior. Instead, his perspective is that each biological being possesses exactly the type and level of intelligence that is optimal for the ecological niche it lives in. In his discussion article, Martin Butz stresses that behavioristic weak AI lacks inductive biases that caused humans and other biological agents to become intelligent beings. He argues that 
such inductive biases and compositional generative models are critical for developing more general strong AI.

\section{Content}

\subsection{Technical Contributions}

- Sensorimotor representation learning for an "active self" in robots: A model survey [4]

Phuong D.H. Nguyen, Yasmin Kim Georgie, Ezgi Kayhan, Manfred Eppe, Verena V. Hafner and Stefan Wermter

- Robot in the mirror: toward an embodied computational model of mirror self-recognition [3]

Matej Hoffman, Shengzhi Wang, Vojtech Outrata, Elisabet Alzueta and Pablo Lanillos

- Goal-directed exploration for learning vowels and syllables: A computational model of speech acquisition [6] Anja Philippsen

\subsection{Al Transfer}

- Prediction error-driven memory consolidation for continual learning. On the case of adaptive greenhouse models [7]

Guido Schillaci, Uwe Schmidt and Luis Miranda

\subsection{Project Reports}

- Robots learn complexity of tasks and actions in automatic curriculum learning: Domain knowledge by hierarchical reinforcement learning, emergence of affordances and active imitation learning [5]

Sao Mai Nguyen, Nicolas Duminy, Alexandre Manoury, Dominique Duhaut and Cedric Buche

\subsection{Discussions}

- Towards strong AI [1]

Martin V. Butz

\subsection{Interviews}

- Intelligent behavior depends on the ecological niche [2] Manfred Eppe and Pierre-Yves Oudeyer

\section{Service}

\subsection{Conferences and Workshops}

- International Conference on Development and Learning and Epigenetic Robotics (ICDL-Epirob), https://icdl2021.org/
- Annual Conference of the Cognitive Science Society (CogSci) https://cognitivesciencesociety.org/future-confe rences/

- International Conference on Intelligent Robots and Systems (IROS), https://www.iros2022.org/index.html

- International Conference on Artificial Neural Networks (ICANN), https://e-nns.org/icanns/

- Conference on Cognitive Computational Neuroscience (CNN), https://ccneuro.org/2020/.

- Conference on Robot Learning (CoRL), https://www. robot-learning.org/.

- Artificial Intelligence and Cognition (AIC). International Workshop Series, http://www.aicworkshopseries.org/.

\subsection{Journals}

- Transactions on Cognitive and Developmental Systems, https://cis.ieee.org/publications/t-cognitive-and-devel opmental-systems

- Trends in Cognitive Sciences, https://www.cell.com/ trends/cognitive-sciences/home

- Robotics and Autonomous Systems, https://www.journ als.elsevier.com/robotics-and-autonomous-systems

- Journal of Intelligent and Robotic Systems, https://www. springer.com/journal/10846

- Adaptive Behavior, https://journals.sagepub.com/home/ $\mathrm{adb}$

- Frontiers in Neurorobotics, https://www.frontiersin.org/ journals/neurorobotics

- International Journal of Humanoid Robotics, https:// www.worldscientific.com/worldscinet/ijhr

Funding Open Access funding enabled and organized by Projekt DEAL.

Open Access This article is licensed under a Creative Commons Attribution 4.0 International License, which permits use, sharing, adaptation, distribution and reproduction in any medium or format, as long as you give appropriate credit to the original author(s) and the source, provide a link to the Creative Commons licence, and indicate if changes were made. The images or other third party material in this article are included in the article's Creative Commons licence, unless indicated otherwise in a credit line to the material. If material is not included in the article's Creative Commons licence and your intended use is not permitted by statutory regulation or exceeds the permitted use, you will need to obtain permission directly from the copyright holder. To view a copy of this licence, visit http://creativecommons.org/licenses/by/4.0/.

\section{References}

1. Butz MV (2021) Towards strong AI. KI-Künstliche Intell. https:// doi.org/10.1007/s13218-021-00705-x

2. Eppe M, Oudeyer P-Y (2021) Intelligent behavior depends on the ecological niche. KI-Künstliche Intell. https://doi.org/10.1007/ s13218-020-00696-1 
3. Hoffman M, Wang S, Outrata V, Alzueta E, Lanillos P (2021) Robot in the mirror: toward an embodied computational model of mirror self-recognition. KI-Künstliche Intell. https://doi. org/10.1007/s13218-020-00701-7

4. Nguyen PDH, Georgie YK, Kayhan E, Eppe M, Hafner VV, Stefan Wermter (2021) Sensorimotor representation learning for an "active self" in robots: a model survey. KI-Künstliche Intell. https ://doi.org/10.1007/s13218-021-00703-z

5. Nguyen SM, Duminy N, Manoury A, Duhaut D, Buche C (2021) Robots Learn complexity of tasks and actions in automatic curriculum learning: domain knowledge by hierarchical reinforcement learning, emergence of affordances and active imitation learning. KI-Künstliche Intell. https://doi.org/10.1007/s13218-021-00708 $-8$

6. Philippsen A (2021) Goal-directed exploration for learning vowels and syllables: a computational model of speech acquisition. KIKünstliche Intell. https://doi.org/10.1007/s13218-021-00704-y

7. Schillaci G, Schmidt U, Miranda L (2021) Adaptation to novel visuo-motor transformations: further evidence of functional haptic neglect. KI-Künstliche Intell. https://doi.org/10.1007/s13218-02000700-8 